\title{
Nonextensive statistical mechanics and economics
}

\author{
Constantino Tsallis and Celia Anteneodo \\ Centro Brasileiro de Pesquisas Físicas \\ Rua Xavier Sigaud 150, 22290-180 Rio de Janeiro, RJ, Brazil \\ Lisa Borland and Roberto Osorio * \\ Evnine-Vaughan Associates, Inc. \\ 456 Montgomery Street, Suite 800, San Francisco, CA 94104-1279, USA
}

(October 23, 2018)

\begin{abstract}
Ergodicity, this is to say, dynamics whose time averages coincide with ensemble averages, naturally leads to Boltzmann-Gibbs (BG) statistical mechanics, hence to standard thermodynamics. This formalism has been at the basis of an enormous success in describing, among others, the particular stationary state corresponding to thermal equilibrium. There are, however, vast classes of complex systems which accomodate quite badly, or even not at all, within the BG formalism. Such dynamical systems exhibit, in one way or another, nonergodic aspects. In order to be able to theoretically study at least some of these systems, a formalism was proposed 14 years ago, which is sometimes referred to as nonextensive statistical mechanics. We briefly introduce this formalism, its foundations and applications. Furthermore, we provide some bridging to important economical phenomena, such as option pricing, return and volume distributions observed in the financial markets, and the fascinating and ubiquitous concept of risk aversion. One may summarize the whole approach by saying that BG statistical mechanics is based on the entropy $S_{B G}=-k \sum_{i} p_{i} \ln p_{i}$, and typically provides exponential laws for describing stationary states and basic time-dependent phenomena, while nonextensive statistical mechanics is instead based on the entropic form $S_{q}=k\left(1-\sum_{i} p_{i}^{q}\right) /(q-1)$ (with $\left.S_{1}=S_{B G}\right)$, and typically provides, for the same type of description, (asymptotic) power laws.
\end{abstract}

Connections between dynamics and thermodynamics are far from being completely elucidated. Frequently, statistical mechanics is presented as a self-contained body, which could dispense dynamics from its formulation. This is an unfounded assumption (see, for instance, [1] and references therein). Questions still remain open even for one of its most well established equilibrium concepts, namely the Boltzmann-Gibbs (BG) factor $e^{-E_{i} / k T}$, where $E_{i}$ is the energy associated with the $i t h$ microscopic state of a conservative Hamiltonian system, $k$ is Boltzmann constant, and $T$ the absolute temperature. For example, no theorem exists stating the necessary and sufficient conditions for the use of this celebrated and ubiquitous factor to be justified. In the mathematician F. Takens' words [2]:

The values of $p_{i}$ are determined by the following dogma: if the energy of the system in the $i^{\text {th }}$ state is $E_{i}$ and if the temperature of the system is $T$ then: $p_{i}=\exp \left\{-E_{i} / k T\right\} / Z(T)$, where $Z(T)=\sum_{i} \exp \left\{-E_{i} / k T\right\}$, (this last constant is taken so that $\left.\sum_{i} p_{i}=1\right)$. This choice of $p_{i}$ is called Gibbs distribution. We shall give no justification for this dogma; even a physicist like Ruelle disposes of this question as "deep and incompletely clarified".

One possible reason for this essential point having been poorly emphasized is that when dealing with short-range interacting systems, BG thermodynamical equilibrium may be formulated without much referring to the underlying dynamics of its constituents. One rarely finds in textbooks much more than a quick mention to ergodicity. A full analysis of the microscopic dynamical requirements for ergodicity to be ensured is still lacking, in spite of the pioneering studies of N. Krylov [3]. In his words:

In the present investigation, the notion of ergodicity is ignored. I reject the ergodical hypothesis completely: it is both insufficient and unnecessary for statistics. I use, as starting point, the notion of motions of the mixing type, and show that the essential mechanical condition for the applicability of statistics consists in the requirement that in the phase space of the system all the regions with a sufficiently large size should vary in the course of time in such a way that while their volume remains constant - according to Liouville's theorem - their parts should be distributed over the whole phase space (more exactly over the layer, corresponding to given values of the single-valued integrals of the motion) with a steadily increasing degree of uniformity. [...] The main condition of mixing, which ensures the fulfilment of this condition, is a sufficiently rapid divergence of the geodetic lines of this Riemann space (that is, of the paths of the system in the $n$-dimensional configuration space), namely, an exponential divergence (cf. Nopf ${ }^{1}$ ).

*tsallis@cbpf.br, celia@cbpf.br, lisa@sphinx.com, roberto@sphinx.com 
Another possibly concomitant reason no doubt is the enormous success, since more than one century, of BG statistical mechanics for very many systems. However, as complex systems become more and more prominent in the front line of research [by complex we mean here the presence of at least one, typically more, of the following features: long-range interparticle interactions, long-term microscopic or mesoscopic memory, (multi)fractal nature of a pertinent subset of phase-space where the system remains long time or forever, small-world or scale-free networking; see [4] for a comprehensive introduction to the subject], this fundamental issue starts being revised (see [5] and references therein).

Indeed, a significant amount of systems, e.g., turbulent fluids ( $[6,7]$ and references therein), electron-positron annihilation [8], cosmic rays [9], economics [10-12], motion of Hydra viridissima [13], kinetic theory [14], classical chaos [15], quantum chaos [16], quantum entanglement [17], long-range-interacting many-body classical Hamiltonian systems ( [18] and references therein), internet dynamics [19], and others, are known nowadays which hardly, or not at all, accomodate within BG statistical mechanical concepts. Systems like these have been successfully handled with the functions and concepts which naturally emerge within nonextensive statistical mechanics [20-22].

The basic building block of nonextensive statistical mechanics is the nonextensive entropy [20]

$$
S_{q} \equiv k \frac{1-\sum_{i=1}^{W} p_{i}^{q}}{q-1} \quad(q \in \mathcal{R})
$$

The entropic index $q$ characterizes the statistics we are dealing with; $q=1$ recovers the usual BG expression, $S_{1}=-k \sum_{i=1}^{W} p_{i} \ln p_{i}$. We may think of $q$ as a biasing parameter: $q<1$ privileges rare events, while $q>1$ privileges common events. Indeed, $0<p<1$ raised to a power $q<1$ yields a value larger than $p$, and the relative increase $p^{q} / p=p^{q-1}$ is a decreasing function of $p$, i.e., values of $p$ closer to 0 (rare events) are benefited. Correspondingly, for $q>1$, values of $p$ closer to 1 (common events) are privileged. Therefore, the BG theory (i.e., $q=1$ ) is the unbiased statistics. A concrete consequence of this is that the BG formalism yields exponential equilibrium distributions (and time behavior of typical relaxation functions), whereas nonextensive statistics yields (asymptotic) power-law distributions (and relaxation functions). Since the BG exponential is recovered as a limiting case, we are talking of a generalization, not an alternative.

The optimization of the entropic form (1) under appropriate constraints [20,21] yields, for the stationary state, the following distribution of probabilities:

$$
p_{i}=\frac{\left[1-(1-q) \beta_{q}\left(E_{i}-U_{q}\right)\right]^{1 /(1-q)}}{Z_{q}}
$$

where

$$
Z_{q} \equiv \sum_{j}\left[1-(1-q) \beta_{q}\left(E_{j}-U_{q}\right)\right]^{1 /(1-q)}
$$

with

$$
U_{q} \equiv \frac{\sum_{j} p_{j}^{q} E_{j}}{\sum_{j} p_{j}^{q}}
$$

and

$$
\beta_{q} \equiv \frac{\beta}{\sum_{j} p_{j}^{q}},
$$

$\beta$ being the optimization Lagrange parameter associated with the generalized internal energy $U_{q}$. Equation (2) can be rewritten as

$$
p_{i} \propto\left[1-(1-q) \beta^{\prime} E_{i}\right]^{1 /(1-q)} \equiv e_{q}^{-\beta^{\prime} E_{i}},
$$

where $\beta^{\prime}$ is a renormalized inverse "temperature", and the q-exponential function is defined as $e_{q}^{x} \equiv[1+(1-$ $q) x]^{1 /(1-q)}=1 /[1-(q-1) x]^{1 /(q-1)}$ (with $e_{1}^{x}=e^{x}$ ). This function replaces, in a vast number of relations and phenomena, the usual BG factor. In particular, the ubiquitous Gaussian distribution $\propto e^{-a x^{2}}$ becomes generalized into the distribution $\propto e_{q}^{-a_{q} x^{2}}=1 /\left[1+(q-1) a_{q} x^{2}\right]^{1 /(q-1)}$ (fat-tailed if $q>1$, and compact support if $q<1$ ).

The use of the concepts and methods of statistical mechanics and thermodynamics in economics has long proved to be a fruitful one ( [23] and references therein). It has even created a specific field of research, whose name - 
Econophysics - has been proposed by Stanley a few years ago. Let us now briefly review three recent applications of the ideas associated with nonextensive statistical mechanics to phenomena in economics, namely a simple trading model which takes into account risk aversion [10], a generalization of the Black-Scholes equation for pricing options [11], and a phenomenological description of distributions of returns and volumes in the real market [12].

\section{Application to risk aversion:}

The works by Kahneman, Tversky and others [24] have put into quantitative evidence the generic and enormous importance of risk aversion (when one expects to win) and risk seeking (when one expects to lose) in trading. The biased averages which naturally emerge in nonextensive statistical mechanics ressemble those proposed in prospect theory, and constitute a tool that can be used to make [10] simple, and relatively realistic, models for a stock exchange or similar forms of trading. In fact, in reference [10], an automaton is introduced which simulates monetary transactions among operators with different attitudes under risky choices. Each operator is characterized by a parameter $q$ which measures his(her) attitude under risk; this index $q$ is the entropic one which plays a central role in nonextensive statistical mechanics. Elementary operations are of the standard type used in hypothetical choice problems that exhibit risk aversion [24]. By following the asset position of the operators, it is possible to conclude on the consequences of each particular attitude on the dynamics of economic operations.

\section{Application to option pricing:}

The celebrated Black-Scholes equation provides, in an explicit manner, the prices of options. Its basic formulation assumes the presence of a Gaussian noise, which simplifies the mathematical treatment on one hand, but yields results which diverge from those observed in real markets. One of us [11] has recently generalized this equation by assuming non-Gaussian fluctuations, evolving anomalously in time according to a nonlinear Fokker-Planck equation. Such a noise process can be modeled as the result of a standard Gaussian process with statistical feedback, of the type discussed in [25]. The degree of feedback is characterized by the nonextensive entropic index $q$; if $q>1$, then rare events will lead to large fluctuations, whereas more common events will result in more moderate fluctuations. This model of stock returns is consistent with empirical observations of the distribution of returns. It is used as a model of stock price fluctuations, from which the fair value of options on the underlying stock can then be derived [11]. The results are appreciably more realistic than the usual Black-Scholes equation which is recovered as the $q=1$ particular case.

This extended model, which preserves the practical advantage of having explicit closed-form solutions, has proved to be a quite satisfactory one. The theoretical option prices predicted by this model, using $q=1.4$ which models well the underlying returns distribution, are in very good agreement with empirically observed option prices. Let us look at the example of European call options. Such options are the right to sell, at expiration time $T$, the underlying stock at an agreed upon price $K$, called the strike price. Whereas the standard Black-Scholes equation must use a different value of the volatilty $\sigma$ for each value of the option strike price in order to produce theoretical values which match empirical ones, the $q=1.4$ model uses just one value of $\sigma$ across all strikes. For the standard Black-Scholes model, a plot of $\sigma$ versus the strike $K$ forms a convex curve known as the volatility smile. We can use the $q=1.4$ model with a fixed $\sigma$ to produce theoretical option prices, and we can then find those $\sigma$ which the standard Black-Scholes model must use in order to produce option prices which match the $q=1.4$ ones. A comparison of such a theoretically obtained volatilty smile with the one observed from market data will reflect how closely the $q=1.4$ model fits real option prices. As can be seen in Figure 1, where the option smile for Japanese Yen futures is studied, there is a very close agreement.

\section{Application to financial returns and volumes:}

In the last few years, the easier availability of databases with detailed transaction histories has enabled an increasing interest in the empirical study of distributions of high-frequency financial variables (see, e.g. [26-30]). We are concerned in this section with two types of single-stock variables defined over fixed time intervals: returns, defined here as logarithmic relative price changes, and volumes, i.e., numbers of shares traded.

When normalized returns (after, for each stock, subtracting their mean and dividing by their standard deviation) are measured over intervals of a few minutes, their distributions are very well fitted by $q$-Gaussians with $q \simeq 1.4$. For long periods (months or years), it seems that values of $q$ that approach the Gaussian limit $(q=1)$ provide an adequate description. Results for high-frequency normalized returns are presented in Figs. 2 and 3 [12] for the 10 top-volume stocks in 2001 in each of the two largest U.S. exchanges: the New York Stock Exchange (NYSE) and the National Association of Security Dealers Automatic Quotation (NASDAQ). 
Similar results are obtained for the corresponding distributions of normalized volumes (divided by their means), by fitting them with $q$-exponentials multiplied by a simple power of the normalized volume. Typical examples are presented in Figs. 4 and 5 [12]. Previous studies have shown that volumes present a power-law behavior at high values [29], while volatilities present both a power-law behavior at high values [26] and a log-normal behavior at smaller values $[26,30]$. Our curves are reminiscent of the behavior of volatility curves, but we suggest, in addition, a new power-law regime at low volumes and propose a functional form that unifies the description of the three regimes. A model whose solution would be this type of distribution would of course be very welcome.

With regard to the specific distributions associated with returns (Figs. 2 and 3), and similar ones ubiquitously observed in nature, a relevant comment is appropriate. The two basic versions of the Central Limit Theorem (CLT) are the following: (1) A convoluted distribution with a finite second moment approaches, in the limit of $N \rightarrow \infty$ convolutions, a Gaussian attractor. (2) A convoluted distribution with a divergent second moment approaches, in the same limit, a Lévy distribution $L_{\gamma}(x)$ (with $2>\gamma>0$ ). If we consider, for example, the $q$-Gaussian distributions indicated above, then (a) if $q<5 / 3$, the attractor is a Gaussian (i.e., $\gamma=2$ ), (b) if $5 / 3<q<3$, the attractor is a Lévy distribution, satisfying $q=(3+\gamma) /(1+\gamma)$, and (c) if $q=5 / 3$ we have a marginal case involving logarithmic corrections. The index $q$ cannot exceed 3 if the norm is to remain finite.

Can we then conclude that all stationary distributions found in nature should be of either the Gaussian or the Lévy type? By no means! It would be so only if the unique dynamics emerging in nature were the convolution dynamics, i.e., with no memory across successive steps. In other words, it would be so if the Laplacian term of the associated Fokker-Planck equation in free space were either a standard second derivative or a fractional derivative. In the first case, the solution is a Gaussian; in the second case (for a derivative of order $\gamma$ ), it is a Lévy distribution. (We assume throughout this discussion the standard first time derivative.)

Actually, much more complex and rich dynamics clearly exist in nature, for example, those associated with a variety of nonlinear Fokker-Planck equations involving nontrivial correlations, multiplicative noise and other effects [31,32], whose exact solutions are $q$-Gaussians.

This remark might seem trivial (it has in fact been already mentioned in [32]), but this point has been overseen by some authors (e.g., [33]). To clarify this, recall that the simple convolution CLT (in both the traditional and the Lévy-Gnedenko versions) only allows, in the large $\mathrm{N}$ limit, for $q=1$ (Gaussians) and $q>5 / 3$ (Lévy distributions). It does not allow in any way for fat-tailed distributions associated with $1<q<5 / 3$. But it happens that, in many cases, complex systems in both nature and the social sciences seem to have a strong "inclination" precisely for that interval! Such is the case for fully developed turbulence in Couette-Taylor experiments [6] (with $1 \leq q \leq 1.2$ for Newtonian turbulence and $q \simeq 1.5$ for Lagrangian turbulence), Hydra viridissima [13] ( $q \simeq 1.5$ ), electron-positron annihilation [8] $(1 \leq q \lesssim 1.3)$, cosmic rays [9] $(q \simeq 1.2)$, and finance [12] $(q \simeq 1.4-1.5)$, among others. There are, however, other situations in nature for which the case is not so clear-cut, although still distinguishable by, e.g., a careful analysis of the behavior near the origin. See Fig. 6 for comparison.

In the specific case of high-frequency financial observations, other studies $[27,28]$ support the facts that (a) variances are finite and (b) the exponent in the power-law tails lies outside the stable Lévy interval $2>\gamma>0$. These observations, together with the obvious presence of long tails, clearly demonstrate the inadequacy of CLT distributions (Lévy or Gaussian) to describe high-frequency returns. In contrast, the use of $q$-Gaussians with $1<q<5 / 3$ is consistent both with finite variances and with the presence of temporal autocorrelations (or memory effects) in the dynamics of financial systems.

\section{ACKNOWLEDGMENTS}

One of us (CT) is very pleased to dedicate his invited talk to his old friend Idahlia Stanley. Two of us (CT and LB) thank Prof. H.E. Stanley and the organizers for their invitation to participate at the Bali meeting. This work has been partially supported by PRONEX/MCT, CNPq, and FAPERJ (Brazilian agencies).

[1] E.G.D. Cohen, Physica A 305, 19 (2002).

[2] F. Takens, in Structures in dynamics - Finite dimensional deterministic studies, eds. H.W. Broer, F. Dumortier, S.J. van Strien and F. Takens (North-Holland, Amsterdam, 1991), page 253. 
[3] N. Krylov, Nature 153, 709 (1944). For full details see N.S. Krylov, Works on the Foundations of Statistical Physics, translated by A.B. Migdal, Ya. G. Sinai and Yu. L. Zeeman, Princeton Series in Physics (Princeton University Press, Princeton, 1979).

[4] M. Gell-Mann, The Quark and the Jaguar: Adventures in the Simple and the Complex (W.H. Freeman, New York, 1999).

[5] M. Baranger, Physica A 305, 27 (2002).

[6] C. Beck, G. S. Lewis and H. L. Swinney, Phys. Rev. E 63, 035303 (2001); C. Beck, Phys. Rev. Lett. 87, 180601 (2001).

[7] T. Arimitsu and N. Arimitsu, Physica A 305, 218 (2002).

[8] I. Bediaga, E. M. F. Curado and J. Miranda, Physica A 286, 156 (2000).

[9] C. Tsallis, J.C. Anjos and E.P. Borges, Fluxes of cosmic rays: A delicately balanced anomalous stationary state, astro$\mathrm{ph} / 0203258(2002)$.

[10] C. Anteneodo, C. Tsallis and A.S. Martinez, Europhys. Lett. 59, 635 (2002).

[11] L. Borland, Phys. Rev. Lett. 89, 098701 (2002); Quantitative Finance 2, 415 (2002).

[12] R. Osorio, L. Borland and C. Tsallis, in Nonextensive Entropy - Interdisciplinary Applications, M. Gell-Mann and C. Tsallis, eds. (Oxford University Press, 2003), in preparation; see also F. Michael and M.D. Johnson, Financial market dynamics, Physica A (2002), in press.

[13] A. Upadhyaya, J.-P. Rieu, J.A. Glazier and Y. Sawada, Physica A 293, 549 (2001).

[14] J. A. S. de Lima, R. Silva and A. R. Plastino, Phys. Rev. Lett. 86, 2938 (2001).

[15] C. Tsallis, A.R. Plastino and W.-M. Zheng, Chaos, Solitons \& Fractals 8, 885 (1997); U.M.S. Costa, M.L. Lyra, A.R. Plastino and C. Tsallis, Phys. Rev. E 56, 245 (1997); M.L. Lyra and C. Tsallis, Phys. Rev. Lett. 80, 53 (1998); U. Tirnakli, C. Tsallis and M.L. Lyra, Eur. Phys. J. B 11, 309 (1999); V. Latora, M. Baranger, A. Rapisarda, C. Tsallis, Phys. Lett. A 273, 97 (2000); F.A.B.F. de Moura, U. Tirnakli, M.L. Lyra, Phys. Rev. E 62, 6361 (2000); U. Tirnakli, G. F. J. Ananos, C. Tsallis, Phys. Lett. A 289, 51 (2001); H. P. de Oliveira, I. D. Soares and E. V. Tonini, Physica A 295, 348 (2001); F. Baldovin and A. Robledo, Europhys. Lett. 60, 518 (2002); F. Baldovin and A. Robledo, Phys. Rev. E 66, 045104(R) (2002); E.P. Borges, C. Tsallis, G.F.J. Ananos and P.M.C. Oliveira, Phys. Rev. Lett. 89, 254103 (2002); U. Tirnakli, Physica A 305, 119 (2002); U. Tirnakli, Phys. Rev. E 66, 066212 (2002).

[16] Y. Weinstein, S. Lloyd and C. Tsallis, Phys. Rev. Lett. 89, 214101 (2002).

[17] S. Abe and A.K. Rajagopal, Physica A 289, 157 (2001), C. Tsallis; S. Lloyd and M. Baranger, Phys. Rev. A 63, 042104 (2001); C. Tsallis, P.W. Lamberti and D. Prato, Physica A 295, 158 (2001); F.C. Alcaraz and C. Tsallis, Phys. Lett. A 301, 105 (2002); C. Tsallis, D. Prato and C. Anteneodo, Eur. Phys. J. B 29, 605 (2002); J. Batle, A.R. Plastino, M. Casas and A. Plastino, Conditional q-entropies and quantum separability: A numerical exploration, quant-ph/0207129 (2002).

[18] C. Anteneodo and C. Tsallis, Phys. Rev. Lett 80, 5313 (1998); V. Latora, A. Rapisarda and C. Tsallis, Phys. Rev. E 64, 056134 (2001); A. Campa, A. Giansanti and D. Moroni, in Non Extensive Statistical Mechanics and Physical Applications, eds. G. Kaniadakis, M. Lissia and A. Rapisarda, Physica A 305, 137 (2002); B.J.C. Cabral and C. Tsallis, Phys. Rev. E 66, 065101(R) (2002); E.P. Borges and C. Tsallis, in Non Extensive Statistical Mechanics and Physical Applications, eds. G. Kaniadakis, M. Lissia and A. Rapisarda, Physica A 305, 148 (2002); A. Campa, A. Giansanti, D. Moroni and C. Tsallis, Phys. Lett. A 286, 251 (2001); M.-C. Firpo and S. Ruffo, J. Phys. A 34, L511 (2001); C. Anteneodo and R.O. Vallejos, Phys. Rev. E 65, 016210 (2002); R.O. Vallejos and C. Anteneodo, Phys. Rev. E 66, 021110 (2002); M.A. Montemurro, F. Tamarit and C. Anteneodo, Aging in an infinite-range Hamiltonian system of coupled rotators, Phys. Rev. E (2003), in press.

[19] S. Abe and N. Suzuki, Itineration of the Internet over nonequilibrium stationary states in Tsallis statistics, Phys. Rev. E (2002), in press.

[20] C. Tsallis, J. Stat. Phys. 52, 479 (1988).

[21] E.M.F. Curado and C. Tsallis, J. Phys. A: Math. Gen. 24, L69 (1991) [Corrigenda: 24, 3187 (1991) and 25, 1019 (1992)]; C. Tsallis, R.S. Mendes and A.R. Plastino, Physica A 261, 534 (1998).

[22] S.R.A. Salinas and C. Tsallis, eds., Nonextensive Statistical Mechanics and Thermodynamics, Braz. J. Phys. 29, No. 1 (1999); S. Abe and Y. Okamoto, eds., Nonextensive Statistical Mechanics and its Applications, Series Lecture Notes in Physics (Springer-Verlag, Berlin, 2001); G. Kaniadakis, M. Lissia and A. Rapisarda, eds., Non Extensive Statistical Mechanics and Physical Applications, Physica A 305, No 1/2 (Elsevier, Amsterdam, 2002); M. Gell-Mann and C. Tsallis, eds., Nonextensive Entropy - Interdisciplinary Applications (Oxford University Press, 2003), in preparation; H.L. Swinney and C. Tsallis, eds., Anomalous Distributions, Nonlinear Dynamics, and Nonextensivity, Physica D (2003), in preparation. An updated bibliography can be found at the web site http://tsallis.cat.cbpf.br/biblio.htm

[23] R. N. Mantegna and H. E. Stanley, Nature 376, 46 (1995); R. N. Mantegna and H. E. Stanley, Introduction to Econophysics: Correlations $\&$ Complexity in Finance (Cambridge University Press, Cambridge, 2000); H. E. Stanley, L. A. N. Amaral, S. V. Buldyrev, P. Gopikrishnan, V. Plerou, and M. A. Salinger, in Proc. Natl. Acad. Sci. Arthur M. Sackler Colloquium, Self-Organized Complexity in the Physical, Biological, and Social Sciences (23-24 March 2001, Beckman Center, Irvine, CA), Proc. Natl. Acad. Sci. 99, Supp.1: 2561 (2002).

[24] D. Kahneman and A. Tversky, Econometrica 47, 263 (1979); A. Tversky and D. Kahneman, Journal of Risk and Uncertainty 5, 297 (1992); A. Tversky and P. Wakker, Econometrica 63, 1255 (1995); A. Tversky and C.R. Fox, Psychological Review 102 (1995) 269; R. Gonzalez and G. Wu, Cognitive Psychology 38, 129 (1999).

[25] L. Borland, Phys. Rev. E 57, 6634 (1998).

[26] Liu, Y., P. Gopikrishnan, P. Cizeau, M. Meyer, C. K. Peng, and H. E. Stanley, Phys. Rev. E 60, 1390 (1999). 
[27] Plerou, V., P. Gopikrishnan, L. A. N. Amaral, M. Meyer, and H. E. Stanley, Phys. Rev. E 60, 6519 (1999).

[28] Gopikrishnan, P., V. Plerou, Y. Liu, L. A. N. Amaral, X. Gabaix, and H. E. Stanley, Physica A 287, 362 (2000).

[29] Gopikrishnan, P., V. Plerou, X. Gabaix, and H. E. Stanley, Phys. Rev. E 62, R4493 (2000).

[30] Andersen, T. G., T. Bollersev, F. X. Diebold, and H. Ebens, J. Financial Economics 63, 43 (2001).

[31] A.R. Plastino and A. Plastino, Physica A 222, 347 (1995); C. Tsallis and D.J. Bukman, Phys. Rev. E 54, R2197 (1996); C. Giordano, A.R. Plastino, M. Casas and A. Plastino, Eur. Phys. J. B 22, 361 (2001); A. Compte and D. Jou, J. Phys. A 29, 4321 (1996); A.R. Plastino, M. Casas and A. Plastino, Physica A 280, 289 (2000); C. Tsallis and E.K. Lenzi, in Strange Kinetics, eds. R. Hilfer et al, Chem. Phys. 284, 341 (2002) [Erratum (2002)]; E.K. Lenzi, L.C. Malacarne, R.S. Mendes and I.T. Pedron, Anomalous diffusion, nonlinear fractional Fokker-Planck equation and solutions, Physica A (2003), in press [cond-mat/0208332]; E.K. Lenzi, C. Anteneodo and L. Borland, Phys. Rev. E 63, 051109 (2001); E.M.F. Curado and F.D. Nobre, Derivation of nolinear Fokker-Planck equations by means of approximations to the master equation, Phys. Rev. E (2003), in press; C. Anteneodo and C. Tsallis, Multiplicative noise: A mechanism leading to nonextensive statistical mechanics, cond-mat/0205314 (2002).

[32] M. Bologna, C. Tsallis and P. Grigolini, Phys. Rev. E 62, 2213 (2000).

[33] D.H. Zanette and M.A. Montemurro, Thermal measurements of stationary nonequilibrium systems: A test for generalized thermostatistics, cond-mat/0212327 (2002).

\section{Captions for figures}

Figure 1: Implied volatilities as a function of the strike price for call options on JY currency futures, traded on May 162002 , with 147 days left to expiration. In this example the current price of a contract on Japanese futures is $\$ 79$, and the risk-free rate of return is $5.5 \%$. Circles correspond to volatilities implied by the market, whereas triangles correspond to volatilities implied by our model with $q=1.4$ and $\sigma=10.2 \%$. The dotted line is a guide to the eye.

Figure 2: Empirical distributions (points) and $q$-Gaussians (solid lines) for normalized returns of the 10 top-volume stocks in the NYSE in 2001. The dotted line is the Gaussian distribution. The 2- and 3-min curves are moved vertically for display purposes.

Figure 3: Same as Fig. 2, but for the NASDAQ.

Figure 4: Empirical distributions (points) and $q$-exponential-like fits (solid lines) for normalized volumes of the 10 topvolume stocks in the NYSE in 2001.

Figure 5: Same as Fig. 4, but for the NASDAQ.

Figure 6: Lévy distributions $L_{\gamma}(x) \equiv \frac{1}{2 \pi} \int_{-\infty}^{\infty} \mathrm{d} k \cos k x \mathrm{e}^{-\alpha|k|^{\gamma}}$, with $0<\gamma<2$ and $\alpha>0$ (black curves), and $q$-Gaussians $P_{q}(x) \equiv\left[1-(1-q) \beta x^{2}\right]^{1 /(1-q)} / Z_{q}$, with $5 / 3<q<3, \beta>0$ and $Z_{q}=\sqrt{\frac{\pi}{\beta(q-1)}} \Gamma\left(\frac{3-q}{2(q-1)}\right) / \Gamma\left(\frac{1}{q-1}\right)$ (red curves). Parameters $(q, \gamma)$ are related through $q=\frac{\gamma+3}{\gamma+1}$ so that the tails of both distributions decay with the same power-law exponent. Without loss of generality, we have taken $\beta=1$ which corresponds to a simple rescaling; $\alpha$ was chosen such that $P_{q}(0)=L_{\gamma}(0)$. 


\section{Implied Volatility}

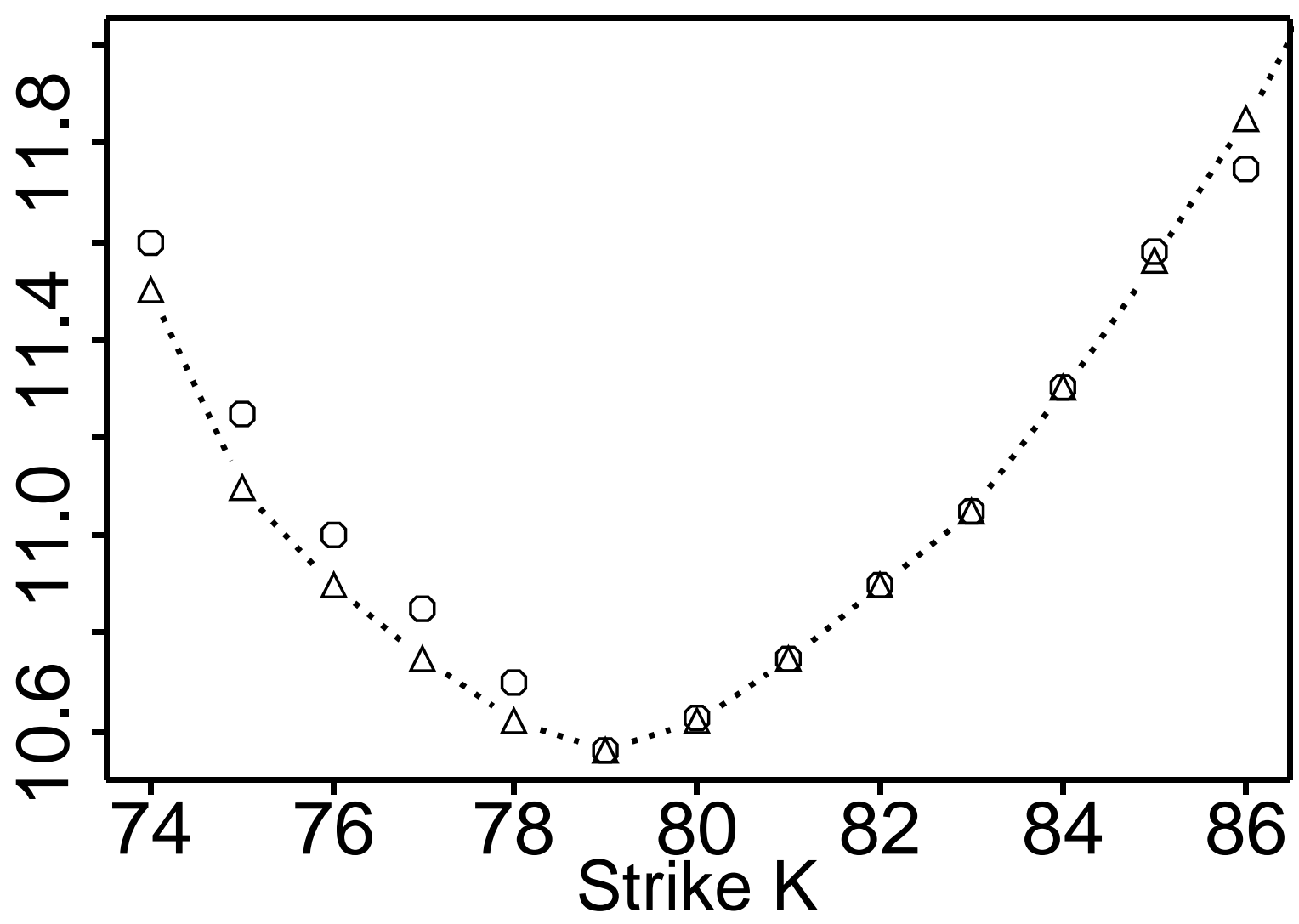


High-Frequency Returns in 2001

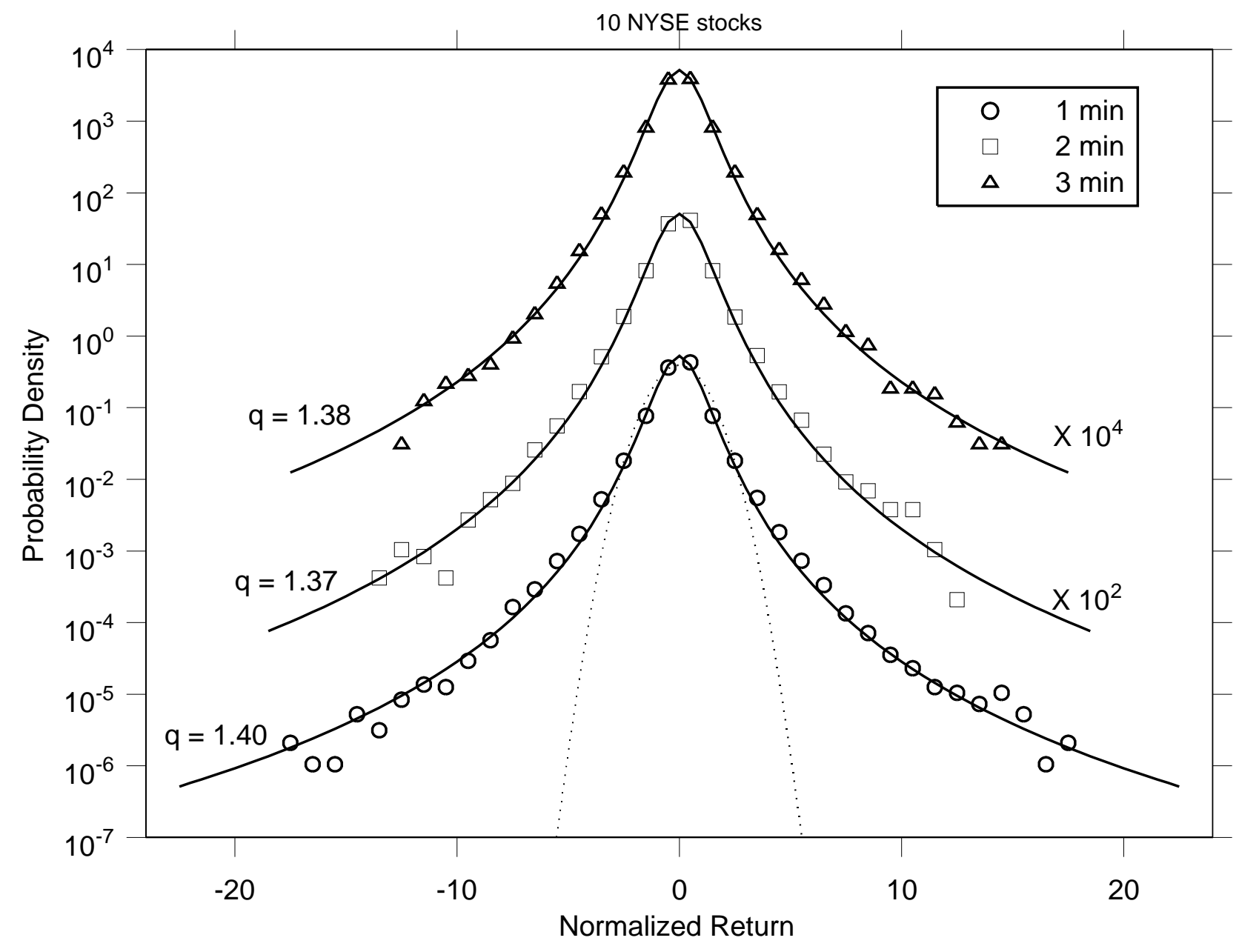


High-Frequency Returns in 2001

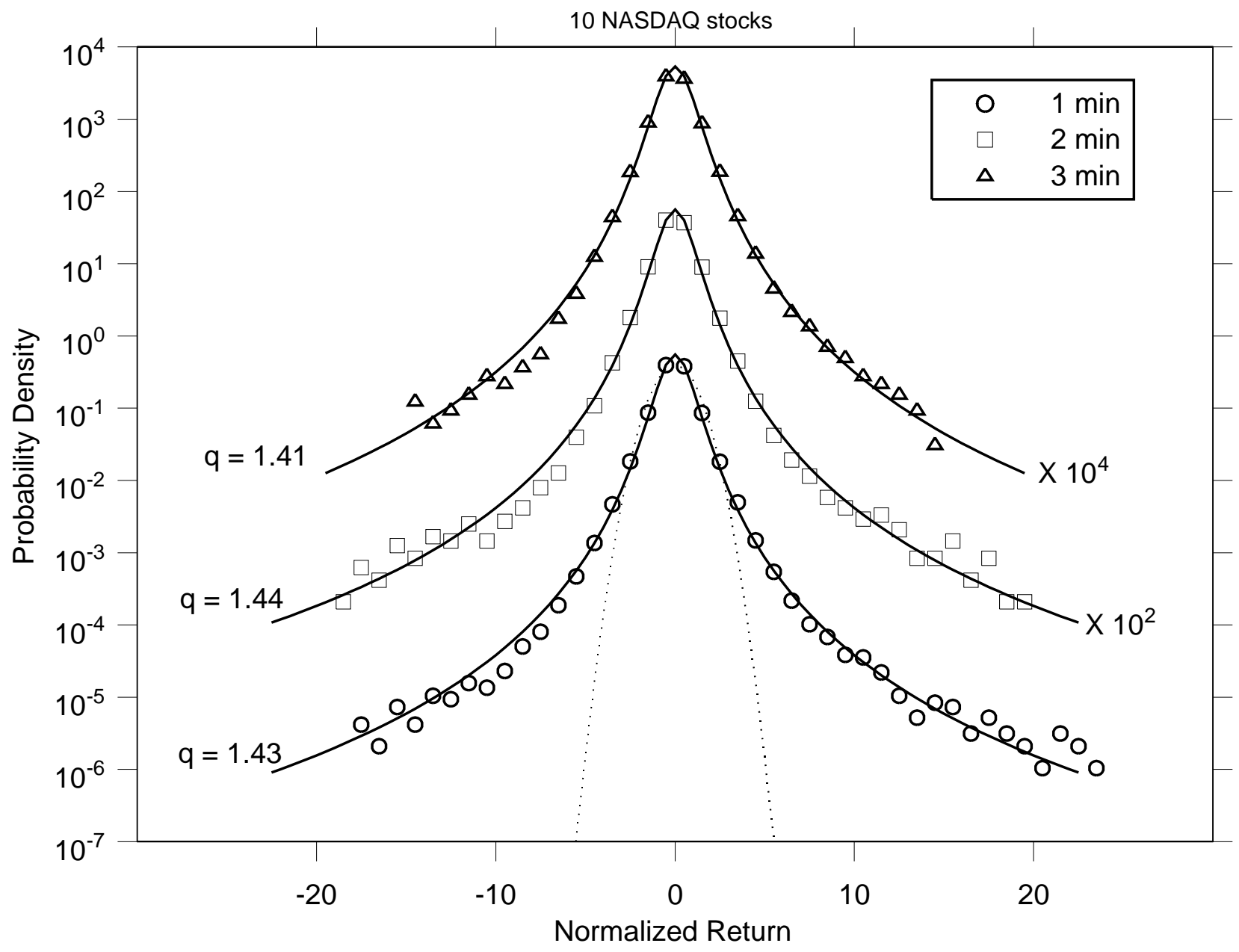


High-Frequency Volumes in 2001

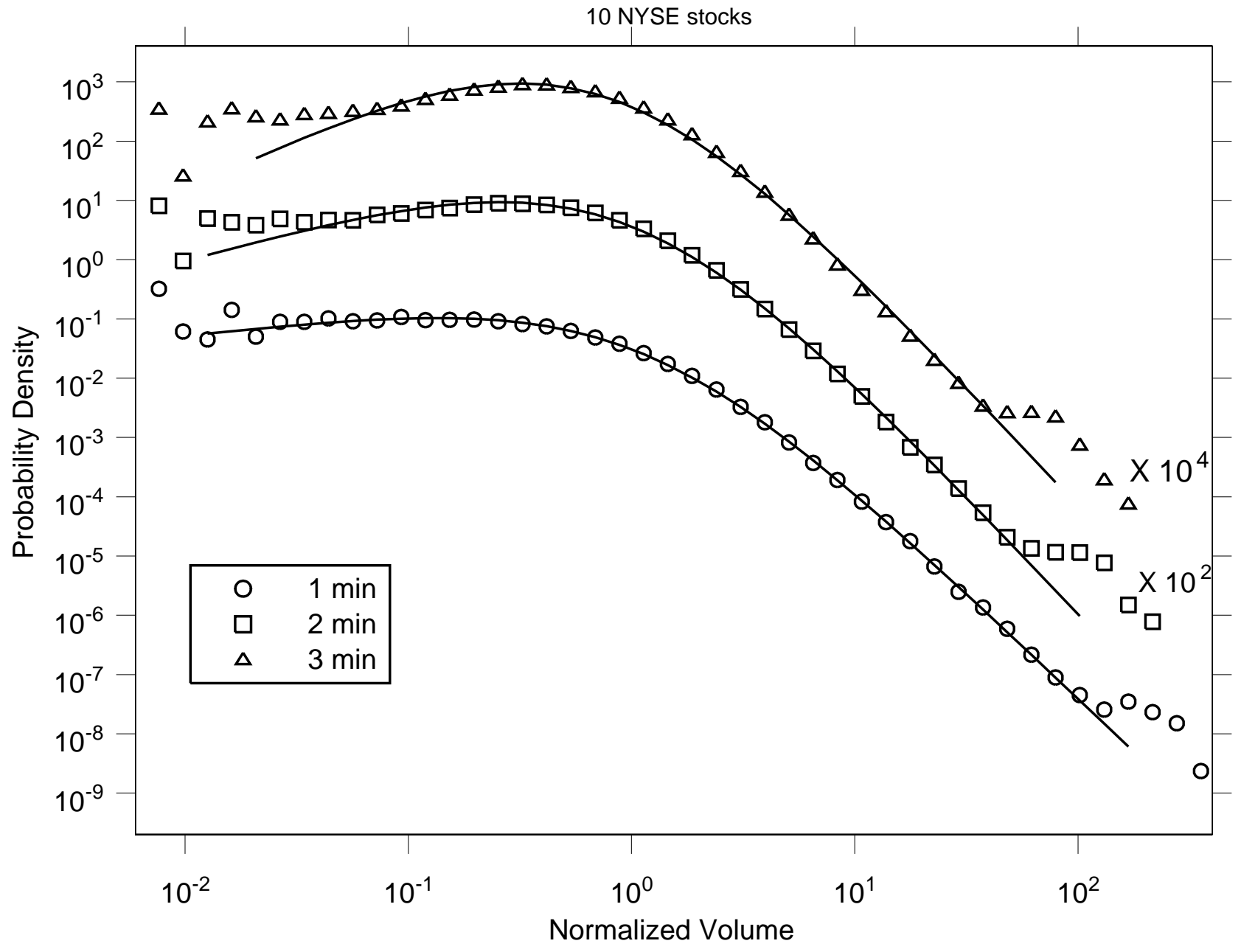


High-Frequency Volumes in 2001

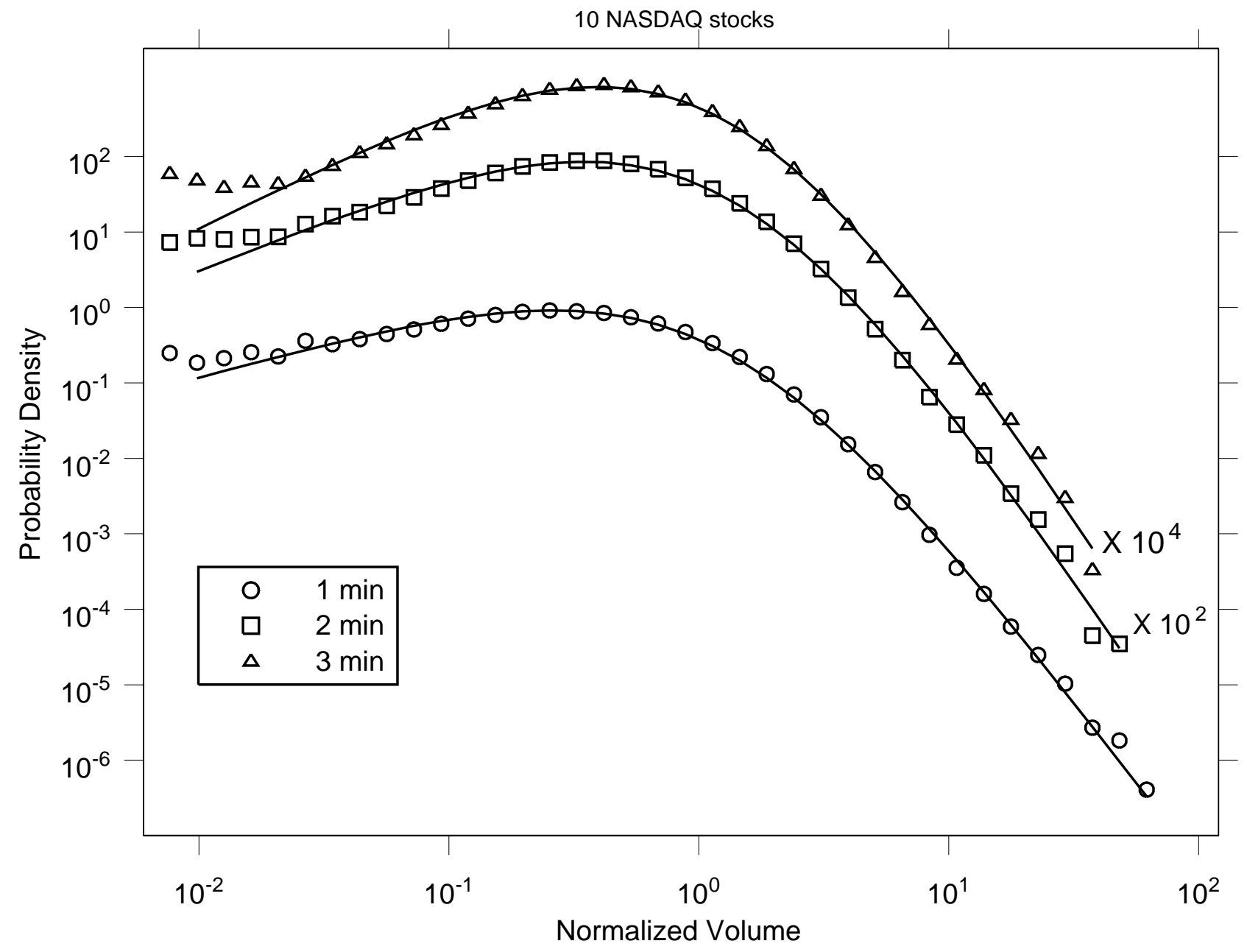



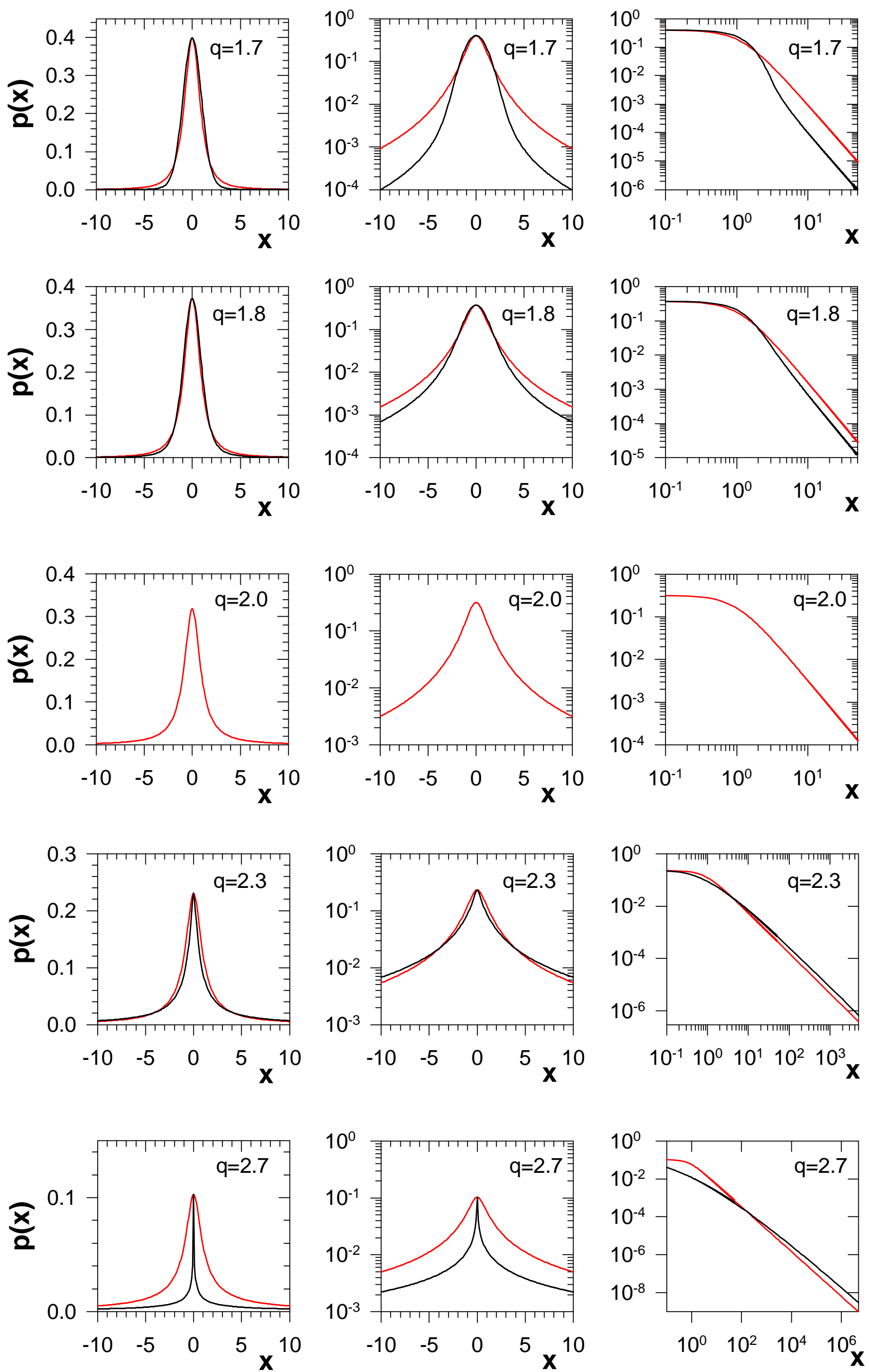